\title{
"Complex trauma": reflections on the effect of early childhood abuse among a small adult group of bariatric patients
}

\author{
Hermann Liebenberg \\ Department of Psychology, University of South Africa, Pretoria, South Africa

\section{Maria Papaikonomou} \\ Department of Psychology, University of South Africa, Pretoria \\ papaim@unisa.ac.za
}

\begin{abstract}
The experience of early traumatic exposure, obesity and finally the paradoxical forced behavioural intervention experienced by a small group of five different participants (three women and two men) who underwent bariatric surgery ${ }^{1}$ to "cure" a chronic disease such as obesity, were explored and structured around the prominent themes identified by the researchers from the "voices" of the participants. The aim was to arrive at a final "voice" that would reflect collectively on their stories. The research question was based on the interrelationship between prolonged traumatic exposures, morbid obesity and whether bariatric surgery acts as a form of remedial intervention for this small group of participants suffering from morbid obesity. A qualitative research paradigm and social constructivist epistemology were supported by the exploratory case study design as a systematic way of collecting data, analysing information through a process of thematic content analysis and finally reporting the results. The delineation of the themes resulted in the following collective sub-themes: Obesity was the result of psychological defences against the impact of prolonged childhood trauma; the symptom constellation of Complex Trauma seems paramount among the participants of this study; and the remedial effect of bariatric surgery on these patients seems complementary to the relief of the impact of early childhood traumatisation. Incidental findings includes: amnesia regarding "good" periods during childhood; obesity creates an "invisible" perception by others of the obese person; to lose weight causes more back pain due to less bodily support by those who had suffered from back pains prior to surgery; while the occurrence of stomach pains after "stressful periods" could be viewed as an unexpected finding from this research group. The research question is based on the interrelationship between prolonged traumatic exposures, morbid obesity and whether the remedial forced behavioural intervention such as bariatric surgery acts as a form of remedial intervention for this small group of participants suffering from morbid obesity.
\end{abstract}

Keywords: Bariatric surgery; Body Mass Index (BMI); Child abuse; Complex Posttraumatic Stress disorder(CPTSD); Complex trauma; Disorder of Extreme Stress not otherwise specified (DESNOS); Early childhood traumatic experiences; Gastric bypass surgery; Morbid obesity; Social constructivism

The terms "Complex Posttraumatic Stress disorder" (CPTSD) or "Disorder of Extreme Stress not otherwise specified" (DESNOS) are perhaps the best known of the non-DSM-IV posttraumatic presentations (Briere, 2004). Disturbances in perception, information processing, affect regulation, impulse control and personality development have up to this point been relegated to various other co-morbidities within the trauma research context (Van der Kolk, 2001). The symptom constellation for "Disorder of Extreme Stress" was used to address the wide array of complexities associated with Complex Trauma in the DSM-IV field trial (1991-1993) (Pelcovitz, Van der Kolk, Roth, Mandel, Kaplan, \& Resick, 1997). "Complex PTSD" is found among people who have been exposed to prolonged traumatic situations, especially during childhood and usually of an interpersonal nature (Herman, 1992a). As suggested by Judith Herman who coined the term Complex Posttraumatic Stress Disorder (CPTSD), children exposed to inescapable stress during childhood will be at risk for compulsivity and re-enactment of trauma by self-abuse syndromes. Herman (1992b) suggests that 
subsequent psychological difficulties are in many cases associated with Axis II disorders.

Van der Kolk (2001) also indicates that the symptom constellation he described as a Disorder of Extreme Stress not otherwise specified (DESNOS) is associated with forms of eating pathology.

Therefore, the term "Complex Trauma" is suggested in order to collectively identify the wide range of symptoms potentially present in traumatised patients, which may include PTSD symptoms as well as symptoms associated with CPTSD and DESNOS.

\section{"COMPLEX TRAUMA", OBESITY AND BARIATRIC SURGERY}

The researchers accept that not all traumatised patients experience eating disorders, however it was observed while dealing with obese patients seeking bariatric surgery ${ }^{1}$ that a typical constellation of symptoms associated with Complex Trauma was more commonly observed among bariatric patients. Eating disorders are determined by a multitude of syndromes and factors, which can be related either directly or indirectly to each other. This article will endeavour to shed some light on the impact of some types of childhood maltreatment by introducing the reader to the proposed link between childhood maltreatment and the symptom constellation known as Complex Trauma within the paradoxical remedial context of bariatric surgery as a positive forced behavioural intervention.

\section{Childhood abuse and Complex Trauma}

As pointed out by Schwartz \& Cohn (1996), supporting Herman (1992a) a specific constellation of symptoms following prolonged childhood trauma together with the inclusion of childhood sexual abuse, maltreatment or inescapable stress, may combine to form syndromes capable of resulting in Complex Trauma. Additionally, seeing that sexual abuse is a form of extreme boundary violation (Schwartz \& Cohn, 1996), it is not surprising that some patients exhibit symptoms of self-injury to the body, of which eating disorders are considered one of those self-inflicting acts. Researchers interpret this extreme form of self-inflicting punishment when sexual abuse has occurred, as the body and sex organs probably becoming the enemy in the distorted survival mechanisms the child uses to maintain the belief that adults are safe and therefore that the body deserves punishment for being bad. More specifically, in the case of females' body size inferences, being feminine in the physical extreme and making the body unacceptable (overweight/obese) may protect the self from the incapacity to say no to male advances. For some women, the internal damages resulting from childhood abuse may cause them to feel that the body becomes the only reason a man would approach them, and that the only way to escape this is to comfort the body from this external threat. It is further concluded that food, in this context, can serve as a transitional object when internal schemas for safety are disrupted.

\section{Obesity as a result of Complex Trauma}

Obesity as an adaptive function to early exposure to trauma was observed by Felitti (2002) who indicated that obesity was possibly a protective mechanism against exposure of childhood trauma. The following statement by Felitti (2002, p. 44) appropriately reflects on the results of this study: "obesity was not their problem; it was their protective solution to problems that previously had never been discussed with anyone".

\section{Bariatric surgery as a remedial intervention}

A comprehensive systematic literature search by Van Hout, Van Oudheusden, and Van Heck (2004) indicated that morbidly obese patients, and specifically those seeking surgical treatment, are described as depressed, anxious, displaying poor impulse control, low self-esteem and impaired quality of life. The typical symptoms and behavioural aspects of obese patients, based on research, indicate a similar constellation to that of some of the symptoms commonly found among patients who present with a complicated form of trauma (Complex Trauma). 
Based on the literature discussed, the researchers concluded that some obese adults who endured prolonged childhood trauma, with certain genetic and psychobiological influences, combined with environmental factors, possess the potential to present with a psychological symptom constellation, known as Complex Trauma. Although the impact of early traumatic exposure as a leading factor in the development and maintenance of obesity is presented as a negative life stressor, it may sometimes facilitate paradoxical positive spin offs, one being surgical interventions. Bariatric surgery, as a forced behavioural intervention not only serves as a remedial mechanism to a life threatening chronic illness and the co-morbid diseases associated with obesity, but also as an emotional lifesaver. Such forced behavioural interventions may protect some individuals from various associated mental illnesses and or emotional distress attributed to the impact of morbid obesity.

\section{METHOD}

\section{Qualitative method within a social constructivist approach}

Within the context of the qualitative research paradigm and the social constructivist epistemology, practical methods were devised to enable the authors to report on the results with regards to the lived experiences of bariatric patients who have had to endure forms of Complex Trauma as children.

\section{Case study design}

The exploratory case study design, which provides a systematic method of looking at events, collecting longitudinal data, analysing information, and reporting the results, was used as the means of inquiry. A hallmark of the exploratory case study design is to condense the case study process, specifically where considerable uncertainty concerning operations, goals and results exists (Creswell, 1998).

To provide a more holistic view of the research participants, the multi-design approach, also called a Patchwork case study (Jensen \& Rodgers, 2001) was applied. This approach was particularly useful for this study because of the diverse nature and quantity of information that needed to be incorporated from among the participants. Multiple methods applied included semi-structured interviews, case notes, clinical observations, questionnaires, collateral information (where available), secondary data analysis and finally thematic content analysis.

\section{Credibility}

Patton (1990) states that credibility depends less on sample size than on the richness of the information gathered and the analytic abilities of the researcher.

The researchers decided to use five subjects for this research and chose participants who are representative of the specific research issues and who could furnish a richness of information related to the aspects under discussion in this study (Patton, 1990).

\section{The participants}

The method used to select the participants was done according to purposive sampling as described by Hoepfl (1997). Purposive sampling, as an approach to an in-depth study, seeks information rich cases (Patton, 1990).

Among the 16 types of purposeful sampling as identified and described by Patton (1990), the researchers decided to apply the principles of "maximum variation sampling" as a specific form of purposive sampling. According to Patton (1990, p. 172), this strategy "aims at capturing and describing the central themes or principal outcomes that cut across a great deal of participant or program variations. For small samples a great deal of heterogeneity can be a problem because individual cases can be different from each other. The maximum variation sampling strategy turns that apparent weakness into strength by applying the following logic: Any common patterns that emerge from great variation are of particular interest and value in capturing the core experiences and central, shared aspects or impacts of a program". 


\section{Selection of participants}

Participants with information-rich voices were approached individually to discuss their willingness to participate in the research. The first author identified potential participants based on the richness of their voices, willingness to participate and the information available from psychometric tests, questionnaires and case notes.

Potential participants were identified that present with symptoms of Complex Trauma, and were approached individually, to discuss their willingness to take part in the research as proposed. Participants included in the study had to fit the following basic criteria:

- Adult males and females (not younger than 18 years);

- Bariatric patients who already underwent bariatric surgery and had the ability to provide detailed, rich information about their experiences;

- Only patients with a Body Mass Index Index (BMI) ${ }^{2}$ of 35 and more, thus meeting the criteria for "obesity class II and III", according to the WHO (World Health Organization, 2004), were included.

- Patients with a history of subjection to prolonged (months to years) exposure to totalitarian control or bullying and/or abuse within a traumatic context (childhood and adolescent) were included;

- Willingness to participate in the research and signing of the agreement/declaration was needed to participate in the study.

For ethical reasons the researchers were guided by the code as proposed in the layout of Ethics Relating to Social Science Research with victims of violence and vulnerable groups (Artz \& Lesizwe, 2005) as proposed within the South African context.

The five participants who took part in this research were able to provide the researchers with detailed descriptions (voices) and interpretations of their experiences. The researchers attempted also to include a diversity of participants in terms of cultural background, age, sexual orientation and gender as well as the various forms of observed childhood trauma (sexual, physical, emotional and neglect) to which they had been exposed. Three participants (one middle-aged and married, and two in their thirties and single, named Ann, Ella and Sue $)^{3}$ were white females who had endured prolonged traumatic experiences since childhood. Only one female participant was in therapy prior to the beginning of the process of preparation for bariatric surgery. Two participants (one in his twenties and the other in his thirties, named James and Manny) were a white single male and an Asian married male both of whom had been exposed to childhood trauma.

\section{Data collection}

Hoepfl (1997) refers to interviews and observations as the two prevailing forms of data collection associated with qualitative inquiry, namely primary (in the present study, case notes, written documents, reports, questionnaires and information from the semi-structured interviews prior to bariatric surgery and observations made during therapy) and secondary sources (in this study, case notes made after surgery, collateral information from the participants' support systems and the verbatim transcriptions from the semi-structured interviews subsequent to the bariatric surgery as part of the other information). The researchers focused on these primary and secondary sources of information in order to triangulate the primary and secondary sources of information as well as the objective reflections of a colleague (a clinical psychologist) based on the raw data and the final voices of the five participants collectively.

Among the three types of qualitative interviewing styles that Patton (1990) describes, the researchers chose to focus on the semi-structured interview. Some of the questions were based on the "Structured Interview for Disorders of Extreme Stress (SIDES)" (Pelcovitz et al., 1997) while others focused on observations made during the engagement with the participant pre- and postsurgery. Relevant questions that were not covered during the pre-operative therapeutic engagement 
and aspects that needed clarity in terms of more detailed enquiry were focussed on during the single final semi-structured interview. Participants were interviewed in the first author's therapy rooms, using their preferred language; the duration of the single semi-structured interviews lasted between 60 and 90 minutes. The electronically recorded interviews were later transcribed verbatim as part of the process of thematic content analysis.

\section{Research process}

All the bariatric patients were seen in therapy over a period of six months (three months pre- and three months post-surgery). When the participants were approached to take part in the research they filled in a consent form in which they were made aware of the purposes and functions of the research, their right to participate, their anonymity and the dissemination of the information gained during the research process. The advantages of the consent form were that the participants were made aware of their right to self-determination and informed voluntary consent, their right to confidentiality and anonymity, their right to maintain self-respect and dignity and their right to refuse to participate or withdraw from participation without fear of recrimination. As part of the initial assessment, the aim of the therapeutic involvement was also to determine their suitability for bariatric surgery. Prospective bariatric surgery candidates were also requested to complete a biographical questionnaire (as compiled by the researchers in order to obtain some background information about the prospective candidates to enable the researchers to identify psychological problem areas and to prepare for the first interview).

According to the referring bariatric surgeon all patients were required to comply with all the instructions of his programme in order to be included in the evaluation for bariatric surgery. One of the inclusion criteria is that all bariatric patients must consult with the therapist for at least three sessions pre-surgery and another three sessions post-surgery. The surgeon expects the therapist to conduct these sessions in order to provide him with information in the form of a report as to the patients' psychological risk factors as well as stress moderators and their emotional preparedness to endure surgery and to deal with the emotional demands that follow the physical and emotional adjustments post surgery.

The case notes on each participant as well as the final transcribed interviews were carefully analysed and presented according to themes. The highlighted themes were not necessarily mutually exclusive or the only true reflections represented as an ultimate truth regarding the realities of the experiences of bariatric patients, but constituted global identified themes. The researchers immersed themselves in the collective data (text) of the participants in order to make sense of their experiences of bariatric surgery, based on their case notes and the transcribed interview of each participant. Inferences were drawn from the text and grouped under the various themes.

\section{Analysis}

Thematic content analysis is a technique used for gathering and analysing the content of text. The data analysis focused on the traumatic experiences, obesity and the bariatric surgery. "Other data", not specific to the focus of the inquiry, highlighted aspects not initially obvious to the focus area of the research and added to "unexpected/serendipity findings" that could be considered valuable in future studies.

According to Hoepfl (1997), the analysis process begins with the identification of themes from the raw data, also referred to by Strauss and Corbin (1990) as "open coding", and implies the tentative naming of conceptual categories into which the phenomena under observation will be grouped. The next stage, according to Hoepfl (1997), involves the re-examination of categories to determine how they are linked; this stage is called "axial coding" (Strauss \& Corbin 1990). Finally, the gathered data/content, once thematically analysed, was extrapolated and translated into a story line with the assistance of another colleague (multiple coders) together with reflections of the re- 
searchers' own subjectivity in analysing the data in order to ensure trustworthiness (multiple coders). Although the stages of analysis are described in a linear fashion, they occurred simultaneously and repeatedly.

\section{FINDINGS}

For the purposes of this inquiry the transcribed information after the semi-structured interviews was mapped according to the symptom constellation as it was presented. The main themes are discussed under the following headings: Affect and impulse dysregulation; Alterations in attention or consciousness; Disturbances in self-perception; Disturbances in relationships; Somatisation and Meaning systems.

\section{Affect and impulse dysregulation}

A dominant theme that emerged from all the participants, was the fact that they felt easily upset, overwhelmed, and emotionally sensitive, worried excessively or overreacted to situations with either impulsiveness or anger. According to Ford (1999) and Van der Kolk, Pelcovitz, Roth, Mandel, McFarlane, and Herman (1996), alteration in regulation of affect and impulses as diagnostic criteria for those suffering from Complex Trauma seems typical and is true of the participants in this bariatric group. Food as a common theme among all the participants seemed to be a compensator to " $\mathrm{calm}$ the emotional distress". This tendency manifested when the patients felt emotionally out of control and hence tended to become more compulsive in their behaviour. By implication or direct expression, all the participants experienced a form of depression or anxiety. For example Manny commented: "I try my best not to let it get hold of me, but sometimes it does happen where you lose it, you know? And you can't control it sometimes".

\section{Alterations in attention or consciousness}

All the participants indicated that there were periods in their lives which they could not remember and which could afford an indication of selective amnesia due to traumatic exposure. For Luxenberg, Spinazzola, and Van der Kolk (2001), the chronically traumatised person's ability to seemingly forget certain parts (months or even years) of his/her experiences may be intermittent if confronted with painful emotional reminders of traumatic experiences. Amnesia could not only be a source of distress, but could also complicate history gathering and recall during therapy.

All the respondents indicated directly or by implication that they had or still have feelings of possessing more than one personality. This split in the core personality could be an indication of elements of depersonalisation and transient dissociation. Visual, emotional and somatic elements of an experience which are too overwhelming, may be split off from the traumatised person's own personal narrative and typically be perceptual or sensory, rather than linguistic in nature (Van der Kolk, Van der Hart, \& Marmar, 1996). A unique description used by Manny was that it felt "as if he looked at others through a glass wall" and that even among others and in spite of his body weight, he felt "invisible" at times. This could be interpreted as an almost paradoxical experience of his life world.

\section{Disturbances in self-perception}

A dominant theme amongst the participants after bariatric surgery was that they felt more in control and effective after surgery, which could be an indication of improvement in their self-perception. This is an example of what Sue said: "It happens a lot ... Before the operation my work started to go down ... but I must say it is getting better now".

According to Herman (1992a), descriptions of a negative view of the self due to childhood abuse, led to negative views of being helpless, not understood, damaged, undesirable to others and ineffectual. The presence of these negative views of the self and the world seems evident in the 
descriptions from this group of bariatric patients. To feel permanently damaged due to traumatic exposure as a child, or as a result of the criticism of others because of their body weight, was a dominant theme observed among most of the participants. The complicated deformation of identity therefore presents with a malignant and fragmented sense of the self as contaminated and guilty (Herman, 1992b).

\section{Disturbances in relationships}

For most of the participants, the feeling of being ashamed, different and not understood seems to be overwhelming. Both males indicated that the feelings of shame are associated with feelings of rejection.

Self minimisation of the reality of the true impact of the patient's weight, being overly worried about uncontrollable aspects of life, the inability to be aware of personal safety, and in Ella's case the feelings that she was at "fault" and therefore "deserved" the trauma she had to endure as a child, seem evident in many of the cases. This can be ascribed to the child's perception of being "bad", regardless of the fact that he/she was abused by adults.

The need to avoid others, to be more isolated, and the inability to trust the motives of others also seem to be dominant themes amongst all the participants, as commonly observed among people suffering from Complex Trauma. For all the women who took part in the research, the ability to trust even their intimate partners seemed problematic as Anne observed: "I wonder sometimes if he (husband) loves me. He says he does but ..."

A statement by Herman (1992b, p. 52) that "when trust is lost, traumatized people feel that they belong more to the dead than to the living", seems to describe the feelings of distrust these patients endure. As remarked by Green (1993): "people who endured sexual abuse during childhood have a greater risk for subsequent victimisation during adulthood". This was pertinently evident from the more dominant voices of some female participants in this study. The lack of a healthy reference to healthy interactions, often presents in the form of inappropriate behaviour towards others or allowing others to interact with the self in an inappropriate manner.

\section{Somatisation}

It is not unusual for chronically traumatised patients to present with physical symptoms that often defy medical explanations (Luxenberg, Spinazzola, \& Van der Kolk, 2001). All the participants in this study endured some form of chronic pains for which the treating doctors could not offer a clear explanation. Van der Kolk et al. (1996) pointed out that emotional pain among traumatised individuals, who had lost the ability to put words to their traumatic experiences, could result in physical symptoms as a symbolic way of communicating their inner emotional distress. Obesity is perceived as a symbolic means of trying to express and protect the core person as commonly observed among those suffering from severe forms of obesity. Berkowitz (1998) noticed that traumatised individuals have documented increasing difficulties in their digestive systems, cardiopulmonary and urogenital areas. For the researchers, although not directly reported by the participants, morbid obesity is considered a problem associated with the digestive system. Sue reports an almost paradoxical effect of experiencing stomach pains mostly after stressful periods in her life.

For most of the participants in this study, somatic complaints related to cardiopulmonary symptoms (“... I get chest pains, I used to get them before the operation ..."), were reported. Issues pertaining to their genitalia and/or within a sexual context were reported by all the participants, except Manny. For Ann, her intense pains due to menstruation were relieved after she had bariatric surgery.

Expressions used by the participants that include being "hopeless", "pessimistic", "emptiness", 
"lonely", "distant from others" and "negative about the future" could be indicative of feelings of despair and hopelessness as a common theme amongst this group of patients. Herman's (1992a, 1992b) notion of chronically traumatised individuals who see the world through a very dark lens, who no longer experience having a purpose, and who do not make sense to themselves, is confirmed by the expressions used by this group of bariatric patients as is evident in the following example of James: "I had thoughts of suicide or self-injury. I did not do it but these thoughts came up often". The conclusion derived from their perceptions is that bariatric surgery changes their outlook on life as a whole as may be noted in the following quotation from Ella: "My thoughts were always 'you won't amount to much 'cause you're not worth much'. I feel different now. I can mean something to people".

\section{DISCUSSION}

The major themes that emerged from the participants' stories capture the essence of the crisis they were plunged into once food served as a comforter, eventually resulting in morbid obesity as an adult, to assist in the psychological defences against emotional discomfort due to the impact of childhood trauma. Emotional abuse, feelings of being rejected, being obliged to keep family secrets and the use of food as a comforter and defence mechanism against emotionally painful feelings, as well as the need to be responsible for the wellbeing and care of others, were reported by all the participants.

This study indicated that prolonged exposure to multiple forms of childhood trauma/abuse, inconsistencies, and double standards by parents and/or caregivers imply a double bind/being trapped by a caregiver/s, causing a paradoxical acceptance by the abused person of the capturer/caregiver/s. This, in turn, tended to create confusion with respect to the person's adaptive functioning and feelings of safety as reported among this group of bariatric patients.

Difficulty in expressing or modulating their emotions such as anger, taking excessive risks with respect to their own safety, having suicidal preoccupations, sexual preoccupations or difficulty in modulating sexual impulses according to Felitti, Anda and Nordernberg (1998), seem evident in the dominant themes emanating from this group of bariatric patients' voices. For all the participants, the feelings of anger and the expression thereof, with either physical and/or verbal aggression, passive aggressiveness or destruction, were observed in all the themes.

A dominant description that represents the feelings of disorientation and alterations in attention or consciousness was reported by the majority of the respondents. Variations of descriptions used by the respondents to explain their feelings of transient dissociation included "absent-minded", "lost track of daily activities", disorientation about "places and time" and that the person "felt out of control". According to Draijer and Langeland (1999), experiences that are too overwhelming or traumatic result in the inability to reconcile traumatic experiences and become relegated to separate aspects of consciousness that do not impinge on day-to-day consciousness, as evident from these bariatric patients' voices.

The element of overeating that results in obesity could, by implication or omission, be interpreted as a form of self-destructive behaviour. However, only two of the participants reported either at present, or previously, to have exhibited the intent or attempt to injure themselves when they felt emotionally frustrated. This is a known element of Complex Trauma, according to Luxenberg, Spinazzola, and Van der Kolk (2001).

An attempt to minimise the effect of earlier trauma on the participants' lives, appears to be covered in all the themes. Minimising the true impact of their weight, being overly worried about uncontrollable aspects of life, the inability to be aware of personal safety and, in Ella's case, the feelings that she was at "fault" and therefore "deserved" the trauma she had to endure as a child, seem evident. The child's perception of being bad, regardless of the fact that he/she had been abused by adults, emphatically causes the child to believe that he/she is at fault. These perceptions spring 
directly from the way the child interprets his/her world. The child's pre-operational thinking places $\mathrm{him} / \mathrm{her}$ at the centre of the universe, causing him/her to believe that he/she has caused the abuse (Luxenberg, Spinazzola, \& Van der Kolk, 2001). For Ella, the reality of "not being at fault" as a child, brought a relief once discussed and explained in a therapeutic environment.

This study reveals that adaptation to positive and/or negative life stressors can equally be a major concern for the obese patient. Collectively, all the participants indicated that they expect relief from some medical problems and expect to lead a healthier lifestyle with less medication after surgery. The participants felt that weight loss would improve their capacity to function more efficiently and optimally in their work environments. The following were expectations of the outcome of surgery: the anticipation of being sociable either with close family or others, being more interactive and the expectation of being perceived as different (in terms of altered physique) and "normal" by others. For some, to be regarded as "normal", implies the ability to buy clothes at accessible general clothing stores. Expectations shared by at least two of the participants include having a normal relationship with food and to be more comfortable with aspects of sexual appearance and intimacy. A unique expectation that emerged from Sue's voice was to be more acceptable to the self. This could indicate an expectation that might be related to a more existential need for acceptance and acknowledgement by others. Expectations and fears before and after bariatric surgery with regards to medical issues or complications, work and psychosocial functioning, sexual or intimacy issues, medical and psychological complications, dysfunctional eating patterns, ability to be physically active, self-acceptance (self-image), amount of weight loss before and after surgery, and quality of life are well documented and supported by literature regarding the predictors and outcomes after bariatric surgery. The expectations and fears that this group of bariatric patients expressed are therefore considered synonymous with the described predictors and outcomes associated with bariatric surgery, as described in the literature (Oria \& Moorehead, 1998; Powers, 1992; Powers, 1999; Puhl \& Brownell, 2001; Sarwer, Fabricatore, \& Wadden, 2006; Sogg \& Mori, 2004).

Incidental findings among this group of bariatric patients" "voices" included the array of paradoxical descriptions of, amongst others, amnesia for "good periods"; being morbidly obese but feeling "invisible"; more back pain after surgery due to "less bodily support" as well as more "stomach pains" after "stressful periods". These findings seem unique and unexpected.

\section{STRENGTHS AND LIMITATIONS OF THIS STUDY}

Social constructivist ideas as reflected in this study prevented the dualistic division and evaluation of occurrences in terms of good and bad, implying that aspects like strengths and limitations are context bound. The discussion of specific strengths associated with specific ideas cannot be accepted without questioning and reflecting the exact original ideas from different perspectives.

The flexibility of qualitative methods within the context of social constructivism created an opportunity within which applicable and new ideas can be developed. The researchers attempted to use perceptions from existing resources from their engagement with the participants to create new perceptions, ideas and interpretations as a co-construction of the participants' experiences. A flexible research framework could be confusing to the reader who might have fixed and clearly defined methods of enquiry in mind.

A possible limitation of this study might include the lack of quantifiable methods of inquiry for some, and for others the qualitative nature of this research could prevent the reduction of occurrences to an oversimplified numeric number.

For the researchers, no final and/or ideal interpretation of what is correct or incorrect could be assumed, therefore it was decided to make the research results tentative, negotiable and open to alternative interpretations (Moon, Dillon, \& Sprenkle, 1991). A possible strength of this notion is the possibility for new and hopeful alternative ideas about the research entities, but this could also be limiting in terms of successful or unsuccessful outcomes. 


\section{CONCLUSION}

It can be concluded that this small group of bariatric patients present with aspects of childhood trauma, and therefore may be regarded as presenting with symptoms associated with Complex Trauma. However, the researchers also acknowledge that another researcher might focus on different aspects of the experiences of bariatric patients and that this conclusion was reached on the basis of their perceptions, observations and focus areas of this particular group of bariatric patients. The results from this small group study may serve a purpose and encourage a working hypothesis for other researchers to test in a more quantitative research context, with the purpose of benchmarking some of the patterns observed among this small group of patients, thereby identifying potentially quantifiable trends among bariatric patients.

Based on this small group of participants, the research questions regarding the interrelatedness of childhood trauma, obesity and the positive effect of bariatric surgery are confirmed and could be explored in future studies as a quantitative method to accentuate the true interrelatedness of trauma, obesity and bariatric surgery as a remedial intervention.

\section{NOTES}

1. Bariatric surgery refers to surgical procedures used to alter or remove parts of the stomach or small intestines;

2. Body Mass Index (BMI) or Quetelet Index is a method used to express the relationship between height and weight at any given age, and includes both genders;

3. In all cases, pseudonyms were used to protect the identities of participants.

\section{REFERENCES}

American Psychiatric Association. (2000). Diagnostic and statistical manual of mental disorders (4th edn, text rev.). Washington, DC.

Artz, L., \& Lesizwe, T. (2005). Ethics Relating to Social Science Research with Victims of Violence and Other Vulnerable Groups [Brochure]. Hatfield, South Africa:

Berkowitz, C.D. (1998). Medical consequences of child sexual abuse. Child Abuse and Neglect, 22, 541-550.

Briere, J. (2004). Psychological assessment of adult posttraumatic states: Phenomenology, diagnosis, and measurement (2nd edn). Washington DC, USA: American Psychological Association.

Creswell, J.W. (1998). Qualitative inquiry and Research Design: Choosing among five traditions. London: Sage.

Draijer, N., \& Langeland, W. (1999). Childhood trauma and perceived parental dysfunction in the etiology of dissociative symptoms in psychiatric patients. American Journal of Psychiatry, 156, 379-385.

Felitti, V.J. (2002). The relation between adverse childhood experiences and adult health: Turning gold into lead. Permanente Journal, 6, 44-47.

Felitti, V.J., Anda, R.F., \& Nordernberg, D. (1998). Relationship of childhood abuse to many of the leading causes of death in adults: the adverse childhood experiences (ACE) study. American Journal of Preventative Medicine, 14, 245-258.

Ford, J. (1999). Disorders of Extreme Stress following war-zone military trauma: Associated features of Posttraumatic Stress Disorder or comorbid but distinct syndromes. Journal of Consulting Clinical Psychology, 67, 3-12.

Herman, J.L. (1992a). Complex PTSD: A syndrome in survivors of prolonged and repeated trauma. Journal of Traumatic Stress, 5, 377-391.

Herman, J.L. (1992b). Trauma and Recovery: From Domestic abuse to political terror. London: Pandora.

Hoepfl, M. C. (1997). Choosing qualitative research: A primer for Technology Education researchers. Journal of Technology Education, 9, 1-17.

Jensen, J.L., \& Rodgers, R. (2001). Cumulating the intellectual gold of case study research. Public Administration Review, 61, 236-246.

Luxenberg, T., Spinazzola, J., \& Van der Kolk, B.A. (2001). Complex Trauma and Disorder of Extreme Stress (DESNOS) Diagnosis, Part One: Assessment. Directions in Psychiatry, 21, 373-392.

Moon, S.M., Dillon, D.R., \& Sprenkle, D.H. (1991). On balance and synergy: Family therapy and 
qualitative research revisited. Journal of Marital and Family Therapy, 17, 187-192.

Oria, H.E., \& Moorehead, M.K. (1998). Bariatric Analysis and Reporting Outcome System (BAROS). Obesity Surgery, 8, 487-499.

Patton, M.Q. (1990). Qualitative Evaluation and Research Methods (2nd edn). Newbury Park, CA: Sage.

Pelcovitz, D., Van der Kolk, B.A., Roth, S., Mandel, F., Kaplan, S., \& Resick, P. (1997). Development of a criteria set and a Structured Interview for Disorder of Extreme Stress (SIDES). Journal of Traumatic Stress, 10, 3-16.

Powers, P.S. (1992). Psychiatric issues in bariatric surgery. Obesity Surgery, 2, 315-325.

Powers, P.S. (1999). Eating pathology before and after bariatric surgery: A prospective study. International Journal of Eating Disorders, 25, 293-300.

Puhl, R., \& Brownell, K.D. (2001). Bias, Discrimination, and Obesity. Obesity Research, 9, 788-805.

Sarwer, D.B., Fabricatore, A.N., \& Wadden, T.A. (2006). The behavioral evaluation of bariatric surgery candidates. Obesity Management, 103-109.

Schwartz, M.F., \& Cohn, L. (1996). Sexual abuse and eating disorders. New York: Brunner/ Mazel, Inc.

Sogg, S., \& Mori, D.L. (2004). The Boston Interview for Gastric Bypass: Determining the psychological suitability of surgical candidates. Obesity Surgery, 14, 370-380.

Strauss, A., \& Corbin, J. (1990). Basics of qualitative research: Grounded theory procedures and techniques. Newbury Park, CA: Sage Publications, Inc.

Van der Kolk, B.A. (2001). The assessment and treatment of Complex PTSD. In R. Yehuda (Ed.), Traumatic Stress. New York: American Psychiatric Press.

Van der Kolk, B.A., Pelcovitz, D., Roth, S.H., Mandel, F.S., McFarlane, A., \& Herman, J.L. (1996). Dissociation, somatization, and affect dysregulation: The complexity of adaptation to trauma. American Journal of Psychiatry, 153, 83-93.

Van der Kolk, B.A., Van der Hart, O., \& Marmar, C.R. (1996). Dissociation and information processing in posttraumatic stress disorder. In B.A. van der Kolk, A. MacFarlane, \& L. Weisaeth (Eds), Traumatic Stress: The Effects of Overwhelming Experience on Mind, Body, and Society (pp. 303-327). New York: Guilford Press.

Van Hout, G.C.M., Van Oudheusden, I., \& Van Heck, G.L. (2004). Psychological Profile of the Morbidly Obese. Obesity Surgery, 14, 579-588.

World Health Organization. (2004). Appropriate body-mass index for Asian populations and its implications for policy and intervention strategies. Lancet, 10, 157-163. 